\title{
Flow-Reversal Experiments with Macromolecules to Measure Column End Efficiency and Bed Heterogeneity
}

\author{
Dóra Zelenyánszki ${ }^{1} \cdot$ Adrienn Mester $^{1} \cdot$ Attila Felinger $^{1,2,3}$ (])
}

Received: 10 April 2019 / Revised: 8 June 2019 / Accepted: 24 June 2019 / Published online: 5 July 2019

(c) The Author(s) 2019

\begin{abstract}
A flow-reversal method combined with peak parking has been introduced recently to determine the band broadening occurring at the two respective column ends and in the bed of the packing material. Flow-reversal has a peak compression effect, therefore, the peaks observed are always narrower and more symmetrical than the peaks obtained without reversing the flow. This phenomenon can originate from the compensation of the multipath dispersion effects. In the present study, peak parking and flow-reversal measurements were extended to macromolecules and carried out with human insulin. We observed that the peaks of insulin are always narrower with reversed flow than without reversing the flow, and the compression effect can be significantly larger than it is for small molecules. The contributions of the column inlet and outlet to the total band variance have been characterized.
\end{abstract}

Keywords Peak parking $\cdot$ Arrested flow $\cdot$ Axial heterogeneity $\cdot$ Column end structure $\cdot$ Flow-reversal

\section{Introduction}

In liquid chromatography, flow-reversal-also known as bidirectional flow-measurements have been used for various reasons, for instance for the determination of the local plateheight, for the elution of strongly retained impurities, or for the local characterization of the chemical modification of the stationary phase [1-5].

Recently, Lambert et al. used the peak parking method to measure the effective diffusion coefficient ( $\left.D_{\text {eff }}\right)$ for the estimation of longitudinal band variance combined with the flow-reversal method with an unretained compound (thiourea) to characterize the sample band broadening in the

Dóra Zelenyánszki and Adrienn Mester contributed equally to this work.

Attila Felinger

felinger@ttk.pte.hu

1 Department of Analytical and Environmental Chemistry and Szentágothai Research Center, University of Pécs, Ifjúság útja 6, Pécs 7624, Hungary

2 Institute of Bioanalysis, Medical School, University of Pécs, Szigeti út 12, Pécs 7624, Hungary

3 MTA-PTE Molecular Interactions in Separation Science Research Group, Ifjúság útja 6, Pécs 7624, Hungary chromatographic column and for showing the differences between the two respective column ends [6].

In the aforementioned study, it was observed that flowreversal has a peak compression effect, therefore, the peaks detected after reversing the flow are always narrower and more symmetrical than the peaks obtained without reversing the flow. The origin of this phenomenon is the velocity biases observed in packed columns.

Wong et al. [7] presented results of experiments with downward slurry-packed chromatography columns to measure the axial heterogeneity of the packed bed. They found, that the most poorly packed region of the bed was the column inlet section, irrespective of the bed length.

Gritti et al. [8] designed a custom-made low-dispersion system made of two small-volume optical detection cells placed immediately before and after the column. They applied the flow-reversal technique to determine radial and axial structural heterogeneities of packed chromatographic beds. A rather significant peak refocusing was observed when reversing the flow direction which confirms the existence of long-range velocity biases in packed chromatographic columns.

Gritti and Gilar studied the impact of frit dispersion [9]. They found that in gradient elution chromatography, the outlet frit has a serious limitation on the achieved efficiency. 
In the present study, peak parking and flow-reversal measurements were carried out with a macromolecule, human insulin. A small molecule, such as thiourea can diffuse easily from a fast flow channel to a slow one, however, proteins usually have small diffusivity, thus a different behavior may be expected. When macromolecules are used for peak parking and flow-reversal experiments, increased parking time and rather small flow-rate should be used to study the axial heterogeneity of packed columns.

\section{Theory}

Band variance is defined and used in various units in separation science. The second central moment $\left(\mu_{2}\right)$ of the peaks (band variance) can be measured on time scale, which yields the temporal variance $\left(\sigma_{t}^{2}\right)$. The longitudinal or spatial variance $\left(\sigma_{z}^{2}\right)$ determines the band spreading in the column. It can be calculated from the $\sigma_{t}^{2}$. The extra-column contributions are usually characterized with the volumetric variance $\left(\sigma_{V}^{2}\right)$, which is calculated for a peak as

$\sigma_{V}^{2}=\sigma_{t}^{2} F_{V}^{2}$

It should be noted that the band variances must be corrected for the effect of the extra-column band broadening before the calculations of the flow-reversal experiments. We want to confirm this theory with molecules larger than thiourea, therefore, we used insulin during our measurements. The various contributions to the observed band broadening are thus written as

$\sigma_{t / V}^{2}=\sigma_{\text {system }}^{2}+\sigma_{\text {park }}^{2}+\sigma_{\text {end }}^{2}+\sigma_{\text {bed }}^{2}$

where $\sigma_{\text {park }}^{2}$ is the band broadening originating from the diffusion during the peak parking period, $\sigma_{\text {end }}^{2}$ comes from the inhomogeneity and from the mixing effect at the column ends while $\sigma_{\text {bed }}^{2}$ comes from the broadening of the peak as it passes through the homogeneous column bed. When the moments of the recorded peaks are corrected for the extracolumn broadening, we get the following relationship

$\sigma_{t / V}^{2}=\sigma_{\text {park }}^{2}+\sigma_{\text {end }}^{2}+\sigma_{\text {bed }}^{2}$

As the analyte migrates in the column, its band variance increases linearly with the distance traveled. The longitudinal variance is expressed as

$\sigma_{\text {bed }}^{2}=H z$

The increase of the longitudinal band variance during the parking period $\left(t_{\text {park }}\right)$ can be expressed as follows:

$\sigma_{\text {park }}^{2}=2 D_{\text {eff }} t_{\text {park }}$ where $D_{\text {eff }}$ is the effective diffusion coefficient. When the observed band variance is plotted against the penetration distance, a straight line is obtained, and the local plate height of the homogeneous packing bed is determined from the slope of that line. The intercept of the line gives the combined contributions of peak parking and column ends [6].

$\sigma_{z, \text { app }}^{2}=\sigma_{z, \text { end,app }}^{2}+2 D_{\text {eff }} t_{\text {park }}+2 H z$

\section{Experimental}

\section{Instrument and Chemicals}

Chromatographic analyses were performed with a Shimadzu UFLC XR equipment. The system consisted of solvent delivery units, an automsampler, a column oven, a degasser and an UV-VIS detector (at $220 \mathrm{~nm}$ ), and a computer data station with LCsolution software. In this study, a reversed phase Kinetex $\left(C_{18}\right)$ column $(4.6 \times 100 \mathrm{~mm}$, packed with $2.6 \mu \mathrm{m}$ core-shell particles) was tested, which was provided by Phenomenex (Torrence, CA, USA). During the measurements, the mobile phase was a mixture of acetonitrile and water, both HPLC grade (VWR International), furthermore, trifluoroacetic acid (Sigma Aldrich) additive was used.

The human insulin samples were prepared from analytical standard purchased from Sigma-Aldrich. For the peak parking and flow-reversal experiments, $65 \%$ acetonitrile and 35\% water with $0.1 \%$ trifluoroacetic acid was used as eluent. The flow-rate was set to $0.05 \mathrm{~mL} / \mathrm{min}$, and the column was thermostated at $45^{\circ} \mathrm{C}$. The injection volume was $1.0 \mu \mathrm{L}$, while the concentration of the insulin sample was $0.5 \mathrm{mg} / \mathrm{mL}$.

\section{Taylor-Aris Disperson of Macromolecules}

Since the molecular diffusivity of insulin is about an order of magnitude smaller than that of small molecules, we had to decrease the flow-rate significantly (from $0.2 \mathrm{~mL} / \mathrm{min}$ to $0.05 \mathrm{~mL} / \mathrm{min}$ ), to observe Taylor-Aris dispersion $[10,11]$ of insulin in the connecting capillaries. When the flow-rate is too high, the insulin molecules will have no time sample the entire cross-section of the tubings, thus the extra-column band broadening will be much larger than it should be. The following equation limits the flow velocity when Taylor dispersion should be observed [12]:

$t_{\text {capillary }}<\frac{d^{2}}{D_{m}}$

where $t_{\text {capillary }}$ is the residence time of the analyte in the capillaries, $d$ the diameter of the capillaries and $D_{m}$ the molecular diffusion coefficient [12-14]. On the Shimadzu equipment, the inner diameter of the capillaries is $d=0.18 \mathrm{~mm}$, 
thus $t_{\text {capillary }}<110 \mathrm{~s}$ should hold. Therefore, we established a $F_{v}=0.05 \mathrm{~mL} / \mathrm{min}$ flow-rate with a $120 \mathrm{~s}$ measured extracolumn residence time.

Furthermore, longer parking time periods should be used for peak parking and flow-reversal experiments too. When using insulin, the parking time was at least $60 \mathrm{~min}$, and if larger proteins (for instance BSA) are used, the parking time should be still further increased.

It should also be noted that the principles of spreading of a sample peak in a long straight open tube is studied here. As the tubing becomes shorter than 30 theoretical plates, though, the eluted peak turns into non-gaussian and the theory for long tubes will not apply [15].

\section{Peak Parking Experiments}

Knox and McLaren [16] introduced the peak parking (PP) method, also known as arrested flow method. After the injection of the analyte, the eluent is pumped under isocratic conditions until the sample reaches the middle of the column. Then, the mobile phase flow is arrested for a time called parking time $\left(t_{\text {park }}\right)$, during which the sample band diffuses in the axial direction of the column. When $t_{\text {park }}$ has passed, the elution is resumed until the sample leaves the column.

From the band broadening occurring during $t_{\text {park }}$, the effective axial diffusion coefficient can be calculated. If we plot $\sigma_{z}^{2}$-which is derived from $\mu_{2}$-against $t_{\text {park }}$, a linear correlation is observed. The slope of this straight line gives $D_{\text {eff }}$ based on Eq. $5[17,18]$.

\section{The Flow-Reversal Method}

The flow-reversal method has been used to test the bed heterogeneity at the column ends. The method includes the stopping of the mobile phase flow when an unretained marker has migrated a given penetration distance into the column, and the reversal of the column under the parking time when the flow is arrested. When the flow has been restarted, the sample elutes at the same column end where it entered the column. Due to the specificity of the column packing procedure, one can suppose that the inlet and the outlet ends of the columns represent a different structure and efficiency, hence experiments were performed to test either the column inlet or the outlet, and the $\sigma_{\text {end }}^{2}$ variance was determined based on Eq. 6 [6].

\section{Calculations}

The second central moments of the observed peaks were used for the calculations of variances. The temporal second moments $\left(\sigma_{t}^{2}\right)$ were determined by fitting an exponentially modified Gaussian (EMG) peak to the experimentally recorded peaks. The moments were corrected for the extra-column effect $\left(\sigma_{\text {system }}^{2}=500 \mu \mathrm{L}^{2}\right)$, which was determined by replacing the column with a zero dead-volume union. The variances obtain during the flow-reversal experiments were expressed in $\mu \mathrm{L}^{2}\left(\sigma_{V}^{2}\right)$.

\section{Results and Discussion}

\section{Molecular Diffusivity of Insulin}

There are several empirical equations to estimate the molecular diffusivity of molecules. For globular proteins surrounded with a hydrate shell, the correlation derived by Young et al. can be used [19]:

$D_{m}=8.34 \times 10^{-8} \frac{T}{\eta M_{w}^{1 / 3}}$

where $T$ is the absolute temperature, $\eta$ the viscosity of the mobile phase expressed in $\mathrm{cP}$ and $M_{w}$ is the molecular weight of insulin. For human insulin, this value is $D_{m}=2.94 \times 10^{-6}$ $\mathrm{cm}^{2} / \mathrm{s}$ under the current experimental conditions.

\section{Measurements of $D_{\text {eff }}$ with Peak Parking Method}

The peak parking method permits the measurement the effective molecular diffusion coefficient. After the insulin is injected into the column, the sample zone is arrested in the column, and during the parking time insulin is let free to diffuse. While small molecules, such as thiourea need a short time $(1,2,5,10 \mathrm{~min})$ to diffuse during peak parking experiments, insulin needs much more time to diffuse to a similar extent. Thus, longer parking time periods must be used for both peak parking and flow-reversal experiments. When we work with long parking times $(60,120,180,240$ and $300 \mathrm{~min}$ ) as we can see in Fig. 1, the band broadening of the peaks increases linearly with increasing parking time.

Using the peak parking method, the effective diffusion coefficient of insulin was determined from the slope of the straight line plotted in Fig. 2: $D_{\text {eff }}=1.6 \times 10^{-6} \mathrm{~cm}^{2} / \mathrm{s}$. From this value, we calculated $\sigma_{\text {park }}^{2}=50.3 \mu \mathrm{L}^{2}$ for $t_{\text {park }}=60 \mathrm{~min}$.

\section{Flow-Reversal Experiments}

The column heterogeneity was tested at the respective column ends with flow-reversal. After insulin was injected and the sample zone migrated a given distance into the column $(4,5,6$, or $7 \mathrm{~cm}$ distance from the column inlet or outlet, respectively) the flow of the mobile phase was stopped for $60 \mathrm{~min}$. When the pressure has relaxed, the column was reversed and after a 60 -min parking time the flow was restarted and the sample zone eluted at the same end as it entered the column. 


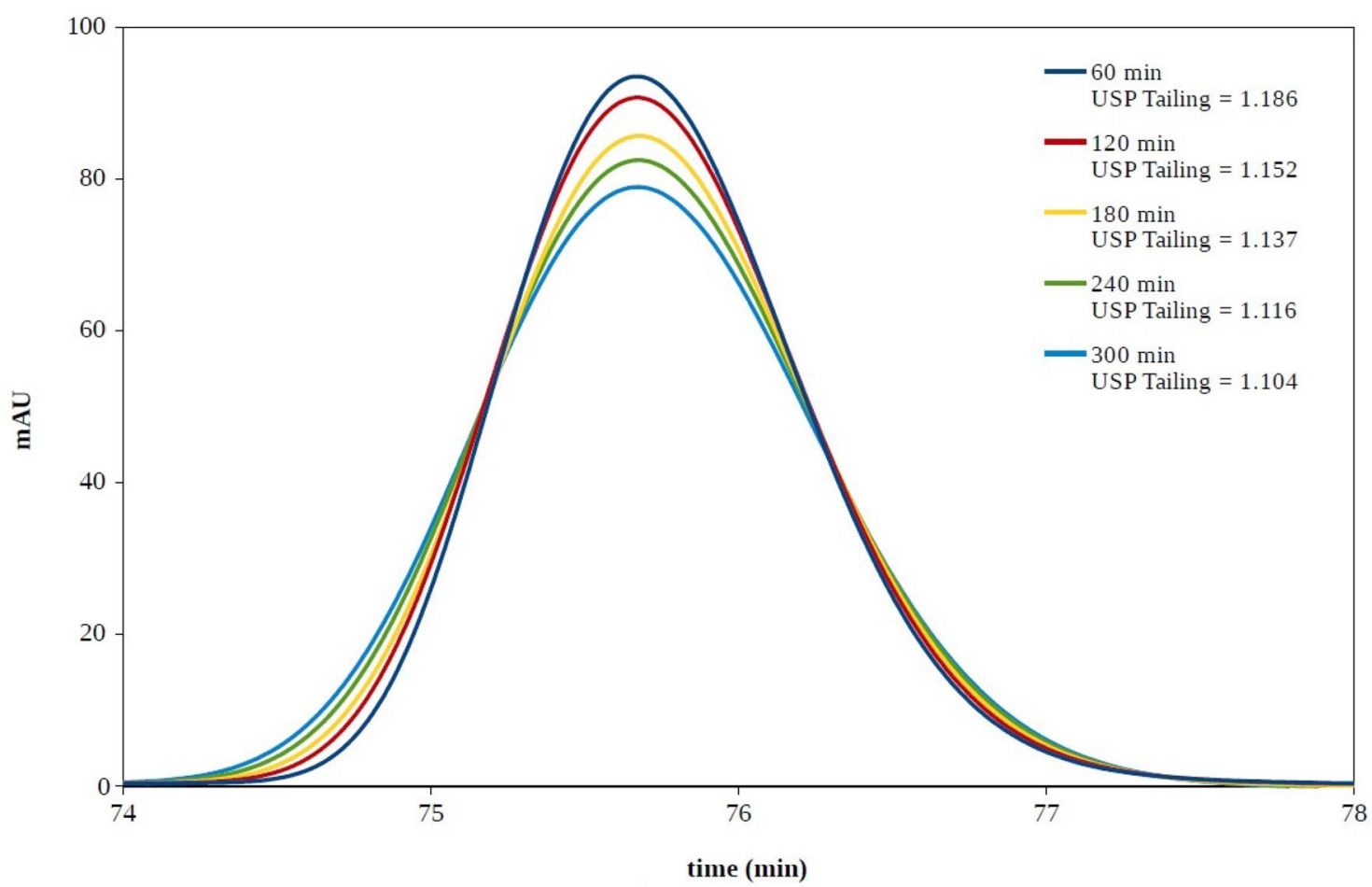

Fig. 1 Peak parking experiments. The peak shapes of insulin observed with the elution in backward flow direction including 60 , 120, 180, 240 and 300 min arrested flow using water:acetonitrile

65:35\% (v/v) as mobile phase at $0.05 \mathrm{~mL} / \mathrm{min}$ flow-rate. The peaks are normalized to 60 min parking time

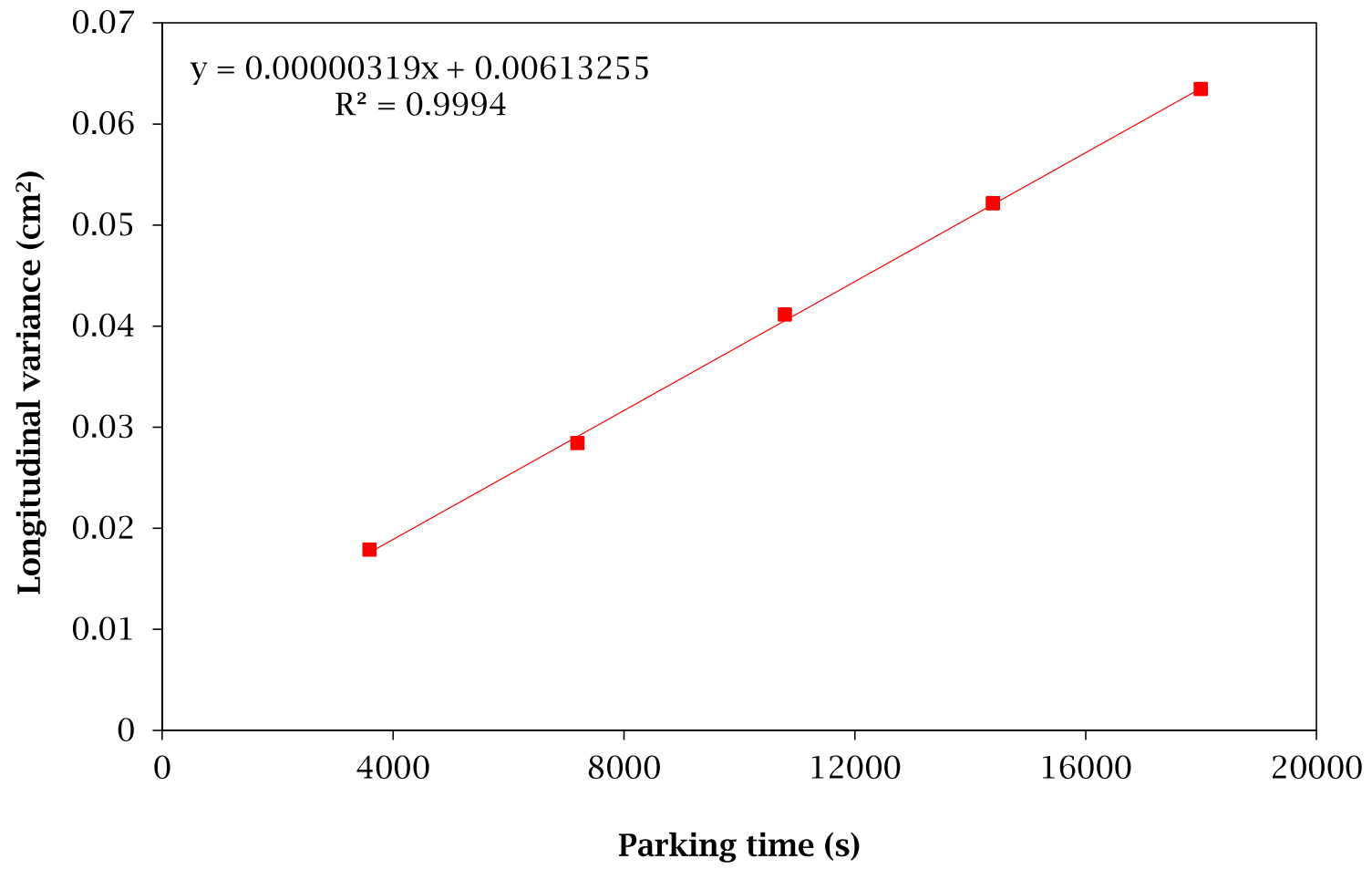

Fig. 2 The change of the longitudinal variance of the insulin peak with increasing parking time. The slope of the fitted straight line gives $2 D_{\text {eff }}$ 
As it can be seen in Fig. 3, with flow-reversal method we noticed slightly narrower and more symmetrical peaks than without reversing the flow and including $t_{\text {park }}=60$ min arrested flow in both cases. Although both peaks are fairly symmetrical in Fig. 3, the USP tailing factor is slightly smaller when the flow is reversed. The peak compression effect due to flow-reversal seems to be small in Fig. 3, but one should note that the relatively large extracolumn peak broadening contribution of the equipment $\left(\sigma_{V, \text { system }}^{2}=500 \mu \mathrm{L}^{2}\right)$ makes the comparison difficult.

The band compression effect of flow-reversal becomes obvious in Fig. 4 where the results have been corrected for the system contribution. If the flow is stopped when the sample zone migrated $5 \mathrm{~cm}$ into the column, the observed variances are much smaller if the flow is reversed after the parking time. For instance, when the column inlet is investigated, the variance is $\sigma_{V}^{2}=139.9 \mu \mathrm{L}^{2}$ if the flow is simply resumed after $t_{\text {park }}=60 \mathrm{~min}$ parking. The variance drops to $\sigma_{V}^{2}=106.7 \mu \mathrm{L}^{2}$ if the flow is reversed before resuming it. In a similar manner, when the column outlet is studied, the variance is $\sigma_{V}^{2}=110.6 \mu \mathrm{L}^{2}$ if the flow is simply resumed after parking and it drops to $\sigma_{V}^{2}=75.6 \mu \mathrm{L}^{2}$ if the flow is reversed before resuming it. These numbers yield $24-32 \%$ focusing effect due to flow-reversal for insulin.
The intrinsic, local plate height for the homogenous column bed was determined from the slope of the fitted straight lines in Fig. 4 using Eq. 3. We found that $H_{\text {forward }}=7.4 \mu \mathrm{m}$ is larger than $H_{\text {backward }}=5.3 \mu \mathrm{m}$. That difference creates a slightly better efficiency when the column is used in the flow direction opposite to what the manufacturer suggests.

From the intercepts of the lines in Fig. 4, we calculated the variances for the column inlet $\left(\sigma_{V \text {,end,in }}^{2}\right)$ and outlet $\left(\sigma_{V, \text { end,out }}^{2}\right)$. The results are summarized in Table 1 as well. There is a difference between the inlet and outlet variances; the inlet end variance is $10 \mu \mathrm{L}^{2}$ larger than the outlet end variance. That difference demonstrates the effect of the column end structure and the difference in packing quality near the respective frits.

When looking at the overall variance, one can state that the homogenous packed bed gives one third and the two column ends the other two thirds of the total band broadening.

\section{Conclusion}

The insulin peaks observed after flow-reversal are definitely narrower and more symmetrical than the peaks obtained without reversing the flow. For small molecules, the band compression effect was found to be less $[6,8]$. The molecular diffusivity of macromolecules is much smaller than that

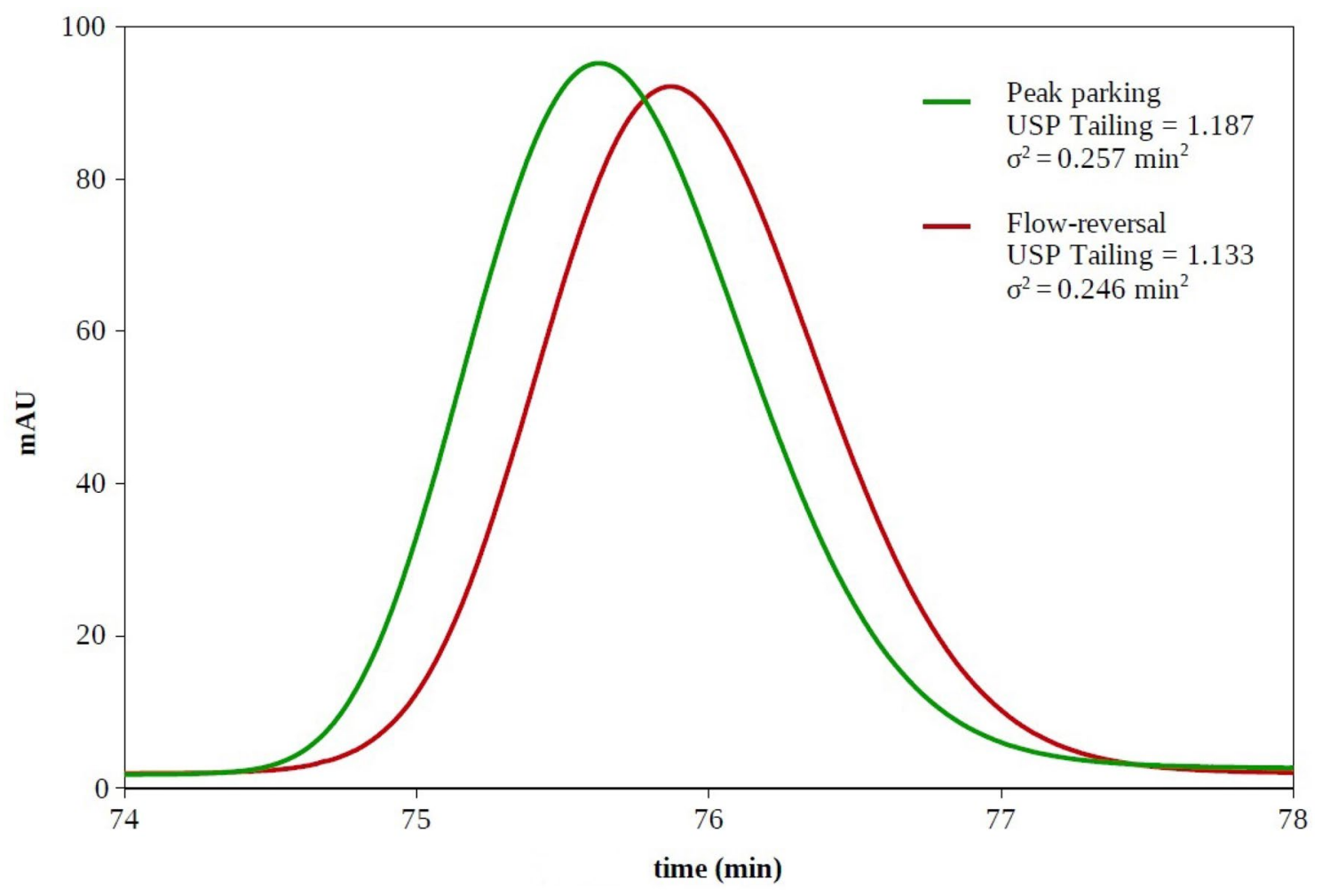

Fig. 3 Comparison of the peak shapes of insulin observed with and without flow-reversal, including 60 min arrested flow in both cases 


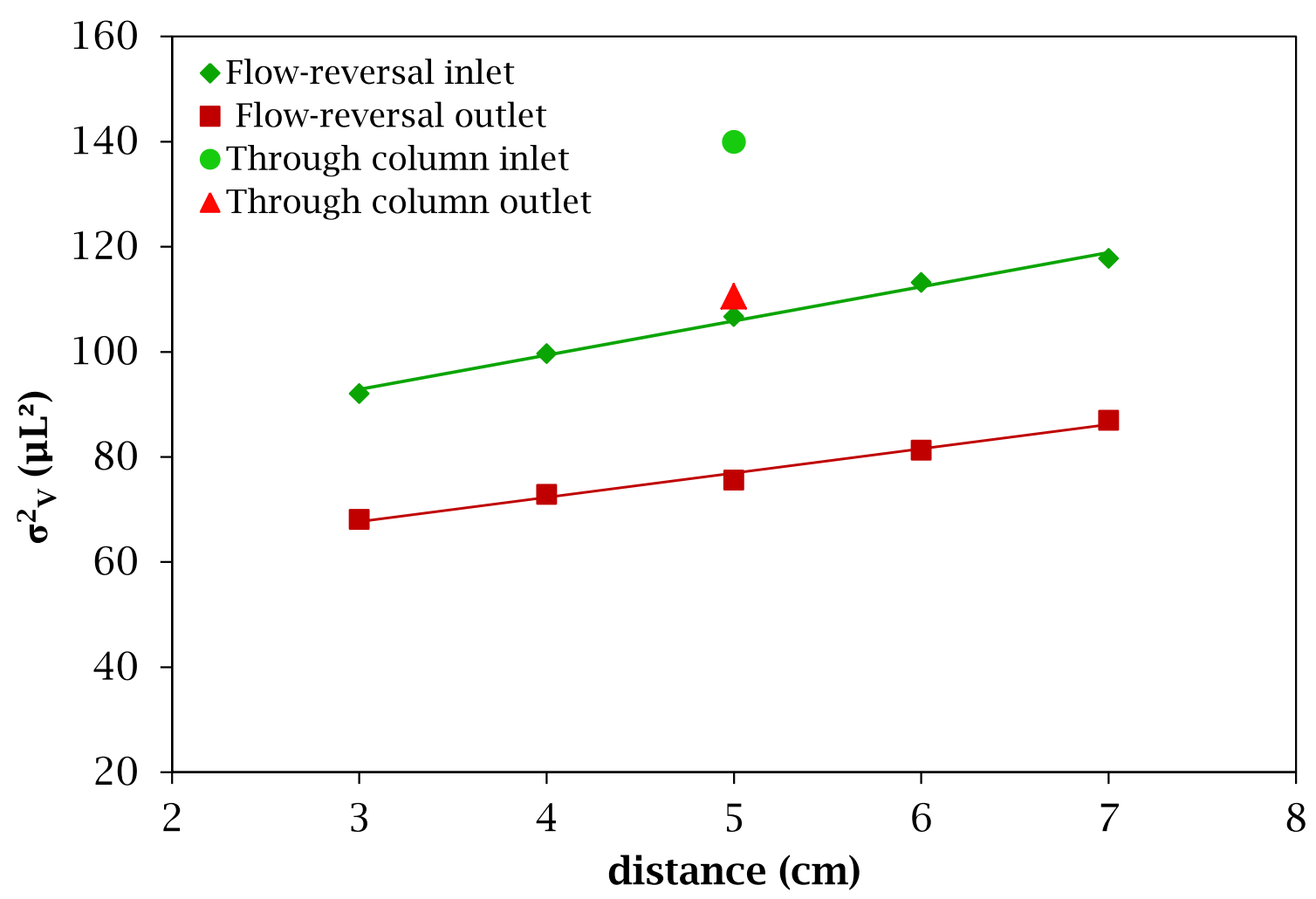

Fig. 4 Volumetric variances obtained with flow-reversal when the sample band was stopped at 3, 4, 5, 6, $7 \mathrm{~cm}$ distance from the column inlet or outlet, respectively. In each case, the flow was arrested for $60 \mathrm{~min}$

Table 1 Volumetric variances obtained for the various band broadening contributions

\begin{tabular}{lll}
\hline & Inlet & Outlet \\
\hline$\sigma_{\text {total }}^{2}$ & $639.9 \mu \mathrm{L}^{2}$ & $610.6 \mu \mathrm{L}^{2}$ \\
$\sigma_{\text {column }}^{2}$ & $89.9 \mu \mathrm{L}^{2}$ & $60.6 \mu \mathrm{L}^{2}$ \\
$\sigma_{V, \text { end }}^{2}$ & $28.5 \mu \mathrm{L}^{2}$ & $18.6 \mu \mathrm{L}^{2}$ \\
$\sigma_{V \text {,bed }}^{2}$ & $32.6 \mu \mathrm{L}^{2}$ & $23.1 \mu \mathrm{L}^{2}$ \\
$H$ & $7.4 \mu \mathrm{m}$ & $5.3 \mu \mathrm{m}$ \\
\hline$\sigma_{V \text {,park }}^{2}=50.3 \mu \mathrm{L}$ & \\
$\sigma_{V, \text { system }}^{2}=500 \mu \mathrm{L}$ &
\end{tabular}

of small molecules. Thus, the radial heterogeneity of the packed bed influences in a lesser extent their migration and consequently a larger band compression effect can observed for macromolecules when the flow is reversed.

With the flow-reversal experiment, we could show the differences between the two respective column ends and also between the local plate heights of the homogeneous column bed when using in forward or backward direction after injecting macromolecules.

For the investigated Kinetex column, the local plate height is somewhat smaller $(5.3 \mu \mathrm{m})$ when we use the column against the recommended flow direction $(7.4 \mu \mathrm{m})$ and the inlet of the column (fitting, frits and column end structure) has a larger contribution to band variance than the outlet of the column. Most probably that difference is due to the column packing procedure, since at the vicinity of the respective column ends the particle density and structure must be different. Furthermore, the band broadening caused by the two column ends is larger than the band broadening in the homogenous bed.

Finally, due to the Taylor-Aris dispersion, the experimental conditions should be significantly altered compared to the studies with small molecules, thus the experiments are much lengthier than with the thiourea or other unretained markers with small molecular weights.

Acknowledgements Open access funding provided by University of Pécs (PTE). This study was funded by the NKFIH OTKA grant K125312. This work was also supported by the UNKP-18-3-I-PTE-125 New National Excellence Program of the Ministry of Human Capacities. The project was also supported by the European Union, cofinanced by the European Social Fund Grant no.: EFOP-3.6.1.-162016-00004 entitled by Comprehensive Development for Implementing Smart Specialization Strategies at the University of Pécs.

\section{Compliance with Ethical Standards}

Conflict of interest The authors declare that they have no conflict of interest. 
Ethical approval This article does not contain any studies with human participants or animals performed by any of the authors.

Open Access This article is distributed under the terms of the Creative Commons Attribution 4.0 International License (http://creativeco mmons.org/licenses/by/4.0/), which permits unrestricted use, distribution, and reproduction in any medium, provided you give appropriate credit to the original author(s) and the source, provide a link to the Creative Commons license, and indicate if changes were made.

\section{References}

1. Eon $\mathrm{CH}$ (1978) Comparison of broadening patterns in regular and radially compressed large-diameter columns. J. Chromatogr. A. 149:29-42

2. Kaminski M (1992) Simple test for determination of the degree of distortion of the liquid-phase flow profile in columns for preparative liquid chromatography. J. Chromatogr. A. 589:61-70

3. Guo H, Frey DD (2010) Interpreting the difference between conventional and bi-directional plate-height measurements in liquid chromatography. J. Chromatogr. A. 1217:6214-6229

4. Roper DK, Lightfoot EN (1995) Estimating plate heights in stacked-membrane chromatography by flow reversal. J. Chromatogr. A. 702:39-80

5. Soliven A, Dennis GR, Hilder EF, Shalliker RA, Stevenson PG (2014) The development of the in situ modification of 1st generation analytical scale silica monoliths. Chromatographia 77:663-671

6. Lambert N, Miyazaki S, Ohira M, Tanaka N, Felinger A (2016) Comparison of the kinetic performance of different columns for fast liquid chromatography, emphasizing the contibutions of column end structure. J. Chromatogr. A. 1473:99-108

7. Wong V, Shalliker R, Guiochon G (2004) Evaluation of the uniformity of analytical-size chromatography columns prepared by the downward packing of particulate slurries. Anal. Chem. 76:2601-2608

8. Gritti F, Dion M, Felinger A, Savaria M (2018) Characterization of radial and axial heterogeneities of chromatographic columns by flow reversal. J. Chromatogr. A 1567:164-176
9. Gritti F, Gilar M (2019) Impact of frit dispersion on gradient performance in high-throughput liquid chromatography. J. Chromatogr. A 76:110-119

10. Taylor G (1953) Dispersion of soluble matter in solvent flowing slowly through a tube. Proc. R. Soc. Lond. A. 219:186-203

11. Aris R (1956) On the dispersion of solute in a fluid flowing through a tube. Proc. R. Soc. Lond. A. 235:67-77

12. Gritti F, Felinger A, Guiochon G (2006) Influence of the errors made in the measurement of the extra-column volume on the accuracies of estimates of the column efficiency and the mass transfer kinetics parameters. J. Chromatogr. A. 1136:57-72

13. Grinias JP, Bunner B, Gilar M, Jorgenson J (2015) Measurement and modeling of extra-column effects due to injection and connections in capillary liquid chromatography. Chromatographia 2:669-690

14. Aggarwal P, Sharma KLS, Lawson JS, Tolley H, Lee ML (2015) Flow rate dependent extra-column variance from injection in capillary liquid chromatography. J. Chromatogr. A. 1380:38-44

15. Atwood JG, Golay MJE (1981) Dispersion of peaks by short straight open tubes in liquid chromatography systems. J. Chromatogr. A 218:97-122

16. Knox JH, McLaren L (1964) A new gas chromatographic method for measuring gaseous diffusion coefficients and obstructive factors. Anal. Chem. 36(8):1477-1482

17. Miyabe K, Ando N, Guiochon G (2009) Peak parking method for measurement of molecular diffusivity in liquid phase system. J. Chromatogr. A. 1216:4377-4382

18. Miyabe K, Nagai J, Guiochon G (2010) Peak parking-moment analysis: a strategy for the measurement of molecular diffusivity in liquid phase. Chem. Eng. Sci. 65:3859-3864

19. Young M, Carroad P, Bell R (1980) Estimation of diffusion coefficients of proteins. Biotechnol. Bioeng. 22:947-955

Publisher's Note Springer Nature remains neutral with regard to jurisdictional claims in published maps and institutional affiliations. 